international cooperation is mandatory. The Japanese Association for Acute Medicine submitted the idea of the JMTDR to the Japanese government. The purpose of JMTDR is to dispatch medical relief teams immediately to disaster-stricken areas in the developing countries, mainly in Asia.

The term "disaster" for JMTDR is limited to natural disasters. This idea was reported by the Ministry of Foreign Affairs at the Cabinet Meeting and was approved and authorized as one of the governmental organizations on March 5th, 1982. Since that time, Japan has sent the JMTDR to six countries, i.e., Ethiopia (Famine), Mexico (Earthquake), Columbia (Eruption of Nevado Del Ruiz and alluvion), Cameroon (Volcanic gas asphyxia), El Salvador (Earthquake), and Solomon (Cyclone).

We analyzed the reports by the team leaders. Each complained of the lack of their own air transport capability. To use Self-Defence Airforce craft to overseas locations is not yet approved by the general public. This restrained us from carrying heavy equipment and vehicles and we were compelled to waste time and bear psychological burdens.

In conclusion, however, JMTDR could help the exhausted local medical staffs in several occasions.

\section{A MAJOR TRAIN ACGIDENT TESTS CONTINGENCY PLANS AND A STATEWIDE EMS SYSTEM}

\section{R Adams Cowley, M.D., William E. Clark, M.S., and Ameen I. Ramzy, M.D., Baltimore, Maryland, U.S.A.}

A sudden crash of a high-speed passenger train into a freight train in Chase, Maryland (U.S.A.) resulted in 16 fatalities and 176 injured persons being treated at hospitals. Another 419 passengers were taken to emergency centers. This mass casualty incident demonstrated the vital importance of having a pre-existing highly coordinated Statewide EMS system. An efficient and effective EMS response was provided by implementing local and state contingency plans. These plans are an extension of normal day-today operational protocols and provide for centralized medical command and control. More than 50 ambulances, several buses and 10 helicopters were used to transport casualties to 11 hospitals for treatment. A precarious stacking of two passenger cars atop a club car presented an exceptionally difficult and unstable situation for rescue and medical personnel who toiled for many hours trying to maintain life and extricate entrapped victims. The last live victim was freed from the wreckage 10-1/2 hours after the crash.

\section{INTERNATIONAL PANEL: RESUSCITATION POTENTIALS IN EARTHQUAKES}

\author{
Peter Safar, M.D., Ernesto Pretto, M.D. and \\ Alberto Villazon, M.D., Pittsburgh, \\ Pennsylvania, U.S.A.
}

Retrospective interviews of surviving lay and physician eye witnesses of the earthquakes in Italy (1980) and Peru (1970) suggest resuscitation could be costeffective (JWAEDM 2:34, 1986). All estimates agreed that about $40 \%$ of those who died slowly and were accessible (about one-third of all killed) might have been resuscitable-if 1) uninjured co-victims had been trained in life-supporting first aid (LSFA); and 2) advanced trauma life support (ATLS) had reached the victims within 6 hours. Potentially reversible slow dying seemed the result of coma (airway obstruction) due to head injury, dust inhalation, external hemorrhage, crushing injuries, internal hemorrhage, and wound infection. Mexico's and other countries' experiences will be summarized.

We intend to invite health professionals who witnessed recent earthquakes in Latin America, to corroborate or modify these impressions, and to explore the possibility of developing international guidelines for resuscitation components within NDMS plans now under development. Estimates of potential benefit and cost are needed for: 1) Mandatory public education in LSFA. 2) Development of novel technologies for prediction and recognition of earthquakes, initiation of response, communication, airlift, finding buried victims, and a traumatic rapid extrication. 3) Medical ATLS teams brought to the scene within 6-12 hours (military). 4) Triage and rapid evacuation to appropriate trauma center with BLS vs. ATLS en route. 5) Field hospitals. 